\title{
Is ultrasonographic evaluation sensitive enough to detect multicentric papillary thyroid carcinoma?
}

\author{
Congqing Lü, Yan Wang", Ming Yu \\ Ultrasound Section, First People's Hospital, Lianyungang 222000, China \\ Contributions: (I) Conception and design: M Yu; (II) Administrative support: M Yu; (III) Provision of study materials or patients: C Lu; (IV) \\ Collection and assembly of data: C Lu; (V) Data analysis and interpretation: All authors; (VI) Manuscript writing: All authors; (VII) Final approval of \\ manuscript: All authors. \\ "These authors contributed equally to this work. \\ Correspondence to: Ming Yu. Ultrasound Section, First People’s Hospital, Lianyungang 222000, China. Email: 497314936@qq.com.
}

\begin{abstract}
Background This study aimed to investigate the preoperative ultrasonographic (US) evaluation of multicentric papillary thyroid carcinoma (PTC) and to evaluate the association of US findings with lymph node metastasis and extracapsular extension in PTC.

Methods: Preoperative US evaluations of patients with PTC who underwent total thyroidectomy were retrospectively investigated. Pathological perspectives and US features of PTC were analyzed. The sensitivity of US in detecting multicentric PTC was evaluated.

Results: The present study included 89 PTC patients who underwent total thyroidectomy. In total, 164 nodules were detected by preoperative US. Significant differences in US pattern were found between benign and malignant nodules. Of the 89 patients with PTC, 33 (37.08\%) cases were confirmed as multicentric PTC by operation and pathological examination, 22 (66.67\%) of which were bilateral. Before surgery, only 23 patients were suspected as multicentric PTC based on US findings. Pathological examination revealed that malignant nodules in 17 (51.51\%) patients with multicentric PTC had been missed by preoperative US. The malignant nodules that went undetected by US were micronodulars (1-4 mm). Furthermore, ultrasonography was less sensitive for the diagnosis of metastatic lymph nodes in the neck. US had more than $80 \%$ sensitivity for detection of extracapsular extension of cases.

Conclusions: US evaluation is not sensitive enough to detect multicentric PTC. The minute size of some nodules in multicentric PTC, may lead to them being missed by US evaluation. Ultrasonography is an optional tool for the detection of extracapsular extension, but it is less sensitive for diagnosing lymph node metastasis.
\end{abstract}

Keywords: Multicentric; papillary thyroid carcinoma (PTC); ultrasonography

Submitted Mar 23, 2020. Accepted for publication Jun 04, 2020.

doi: $10.21037 / g s-20-487$

View this article at: http://dx.doi.org/10.21037/gs-20-487

\section{Introduction}

Over the past two decades, the global incidence of papillary thyroid carcinoma (PTC) has risen $(1,2)$. Ultrasonographic (US) evaluation can provide accurate information about thyroid structures, blood flow, and adjacent tissues, and has therefore been the first-line imaging tool for detecting, stratifying risk, and monitoring treatment of thyroid nodules (3-5). Advances in diagnostic techniques have made it easier to diagnose PTC before thyroidectomy (3-5). Most patients with PTC can expect an excellent prognosis after appropriate therapy (6); however, for a minority, PTC is relatively aggressive, resulting in a poorer prognosis (7). Different variants of PTC have been reported in previous documents (8). The encapsulated follicular variant, the cribriform-morular variant, and the Warthin- 
like variant are associated with favorable outcomes (8). While, tall cell variant, the columnar cell variant, and the hobnail are associated with more unfavorable outcomes (8). The BRAF V600E mutation are closely related to the unfavorable variants (9-11). The solid variant and the diffuse sclerosing variant (DSV) may be associated with less favorable outcomes (8). Radiologists attempt to establish a relationship between ultrasound manifestations and different variants (8-11). Different variants can have the similar ultrasonic performance (8-11).

In addition to the above, different variants and BRAF V600E mutation are associated with the outcome (9-11). In a previous study by Lin et al., patients with multicentric PTC were indicated to have a relatively poor prognosis compared with those with unifocal PTC (12). The study suggested that patients with multicentric papillary thyroid microcarcinoma ( $\mathrm{PTC}<1.0 \mathrm{~cm}$ ) should be categorized as a high-risk group (12). In Lin et al.'s retrospective study (12), a total of 1,682 PTC patients who received total thyroidectomy were analyzed. Of all the cases of PTC, 337 cases $(20.0 \%)$ were multicentric PTC, and $45.8 \%$ of patients with multicentric PTC with five foci experienced cancer recurrence (12). Nodule size is an important factor in choosing treatment for PTC. According to the recommendations of the American Thyroid Association guidelines, low-risk PTC patients with a maximum tumor diameter of $\leq 10 \mathrm{~mm}$ can receive hemithyroidectomy (13). Preoperative detection of multicentric PTC, especially bilateral PTC, may affect the choice of surgeon. In Lin et al.'s study, the size of the largest nodule in patients with multicentric PTC was significantly smaller than that in patients with unifocal PTC (12). The smaller nodule size in PTC increases the difficulty of US evaluation. Because multicentric PTC may be a potential poor predictor (12), patients' outcomes may be improved by preoperative detection of multicentric PTC.

Clinical information on preoperative ultrasound evaluation of multicentric PTC is limited. Previous studies did not described the association of multicentric PTC with variants or mutation $(8,11)$. This study aimed to investigate the preoperative evaluation of multicentric PTC by US. The association between US findings with lymph node metastasis and extracapsular extension in PTC patients who underwent total thyroidectomy was also evaluated.

We present the following article in accordance with the STROBE reporting checklist (available at http://dx.doi. org/10.21037/gs-20-487).

\section{Methods}

\section{Ethical approval}

This study has been approved by Ethics committee of our hospital (No. 2018032). Informed consent was taken from all the patients. The study was conducted in strict accordance with the Declaration of Helsinki of the World Medical Association.

\section{Inclusion and exclusion criteria for patients}

All patients discharged from January 1, 2018 to December 31, 2018 were screened using the hospital's surgical registration system. The medical records of patients who underwent thyroidectomy during the study period were retrospectively reviewed. The inclusion criteria were as follows: (I) patient underwent total thyroidectomy with or without neck lymph node dissection; (II) preoperative ultrasonography was performed in our hospital; and (III). The diagnosis of PTC was confirmed by postoperative histopathological examination. Patients aged $<18$ years or who had missing data were excluded from the analysis. We only chose patients underwent total thyroidectomy in the present study. The results of histopathology can accurately diagnose unifocal PTC or multicentric PTC.

\section{Ultrasonography and Doppler sonography of the thyroid before operation}

Ultrasonography and Doppler sonography of the thyroid were performed by experienced radiologists using a high-frequency (10 MHz or higher) linear probe. The radiologists were fully trained and had obtained professional certifications. The patients were placed in the supine position with maximal tolerated neck extension. Grayscale US evaluation included transverse and longitudinal images of both lobes. The transverse image of the isthmus and the thickness of the isthmus on the transverse view were also evaluated. The imaging features of visible nodules were recorded (3-5). The nodules were graded based on TI-RADS (14). Imaging features of the nodules included size, composition, shape, margins, echogenic foci and calcifications, effect on the thyroid capsule, and/or extension beyond the capsule (3-5). The shape of the nodules was defined as oval, round, nonparallel, or irregular (3). Based on the results of the previous study, a taller-than-wide shape is a highly specific indicator of malignancy (15). The margin 
was classified as well defined or not. The composition of the nodules was classified as cystic, predominantly cystic (cystic portion $>50 \%$ ), predominantly solid (solid portion $>50 \%$ ), solid, or spongiform (3-5). Using adjacent thyroid tissue as the basis for comparison, the echogenicity of each visible nodule was described as hyperechogenic, isoechogenic, or hypoechogenic; however, when markedly hypoechoic nodules were defined, the strap muscles served as the reference (3-5). Calcifications were classified as none, microcalcifications $(\leq 1 \mathrm{~mm})$, macrocalcifications $(>1 \mathrm{~mm})$, or mixed or rim calcifications $(3-5,16,17)$. The following features were defined as extracapsular extension (18): (I) the thyroid nodule seen to abut the capsular margin; (II) bulging of the normal thyroid contour; (III) loss of the echogenic capsule; and (VI) vascularity beyond the capsule, detected by Doppler ultrasonography.

To evaluate tissue adjacent to the thyroid, especially the cervical lymph nodes, US scanning should be extended beyond the thyroid borders (3-5). In the presence of abnormal cervical lymph nodes, imaging features of the nodule were recorded. Doppler imaging of the thyroid was performed after gray-scale ultrasound was completed.

\section{Data collection}

Demographic data including the patient's name, gender, and age were collected. All records of US evaluation were obtained from the hospital's electronic databases. The US evaluations of cases meeting the inclusion criteria were reviewed and the data were collected. The data from postoperative pathological examination were also obtained. Pathological diagnosis information included the diagnosis of thyroid nodules, extension or no extension beyond the capsule, other thyroid diseases, lymph node metastasis or no lymph node metastasis, and missed diagnosis by US.

\section{Data analysis}

The data were input and collected using Microsoft Excel. Data analysis was performed using Statistical Product and Service Solutions (SPSS22.0, IBM). Continuous variables were represented by mean \pm standard deviation for normally distributed variables or by median and interquartile for variables with skewed distributions. The categorical variables were presented as absolute. Benign and malignant nodules were determined based on the pathological findings, and the differences in characteristics between benign and malignant nodules were tested. Based on the characteristics of the variables, Student's $t$-test or Wilcoxon rank-sum test was used for continuous variables and Fisher's exact test was used for categorical variables. A two-tailed $\mathrm{P}$ value $<0.05$ was considered significant.

\section{Results}

From January 1, 2018 to December 31, 2018, 436 patients (>18 years) underwent thyroidectomy in our hospital. Excluding the repeated cases, patients who underwent subtotal thyroidectomy, and patients with incomplete data, 89 patients who underwent total thyroidectomy were included in the present study (Figure S1).

\section{Clinical and US characteristics of 89 patients who underwent total thyroidectomy}

The mean age of the 89 patients who underwent total thyroidectomy was 46 (19-71) years old. Of the included patients, $28(31.46 \%)$ were male and $61(68.54 \%)$ were female. Neck lymph node dissection was received by 81 patients. Before surgery, 164 thyroid nodules were detected by US. US detected 1 thyroid nodule in 21 patients, and $>1$ nodule in 68 patients ( 2 nodules in 61 patients and 3 nodules in 7 patients). Patient clinical and US characteristics are listed in Table 1.

\section{Characteristics and final diagnoses of 164 thyroid nodules detected by preoperative ultrasound}

Of the 164 thyroid nodules, 122 (73.94\%) nodules were malignant. The median size of the thyroid nodules was $9 \mathrm{~mm}$. The difference in median size between the benign nodules and the malignant nodules was not significant. The pathological diagnosis of 42 benign nodules included 32 cases of nodular goiters, 6 cases of nodular goiters with nodular hyperplasia, 2 cases of Hashimoto's thyroiditis, and 2 cases of cystic degeneration of thyroid adenoma. Despite the overlap between some features of benign and malignant thyroid nodules, there were significant differences in US pattern between the benign and malignant thyroid nodules. Twenty-two malignant nodules were taller-than-wide shape. Four malignant nodules were lobulated. In total, six patients were diagnosed as Hashimoto's thyroiditis complicated PTC. In the present study, 83 patients received fine needle aspiration (FNA) puncture before operation. 
Table 1 Clinical and ultrasonographic characteristics of 89 patients who underwent total thyroidectomy

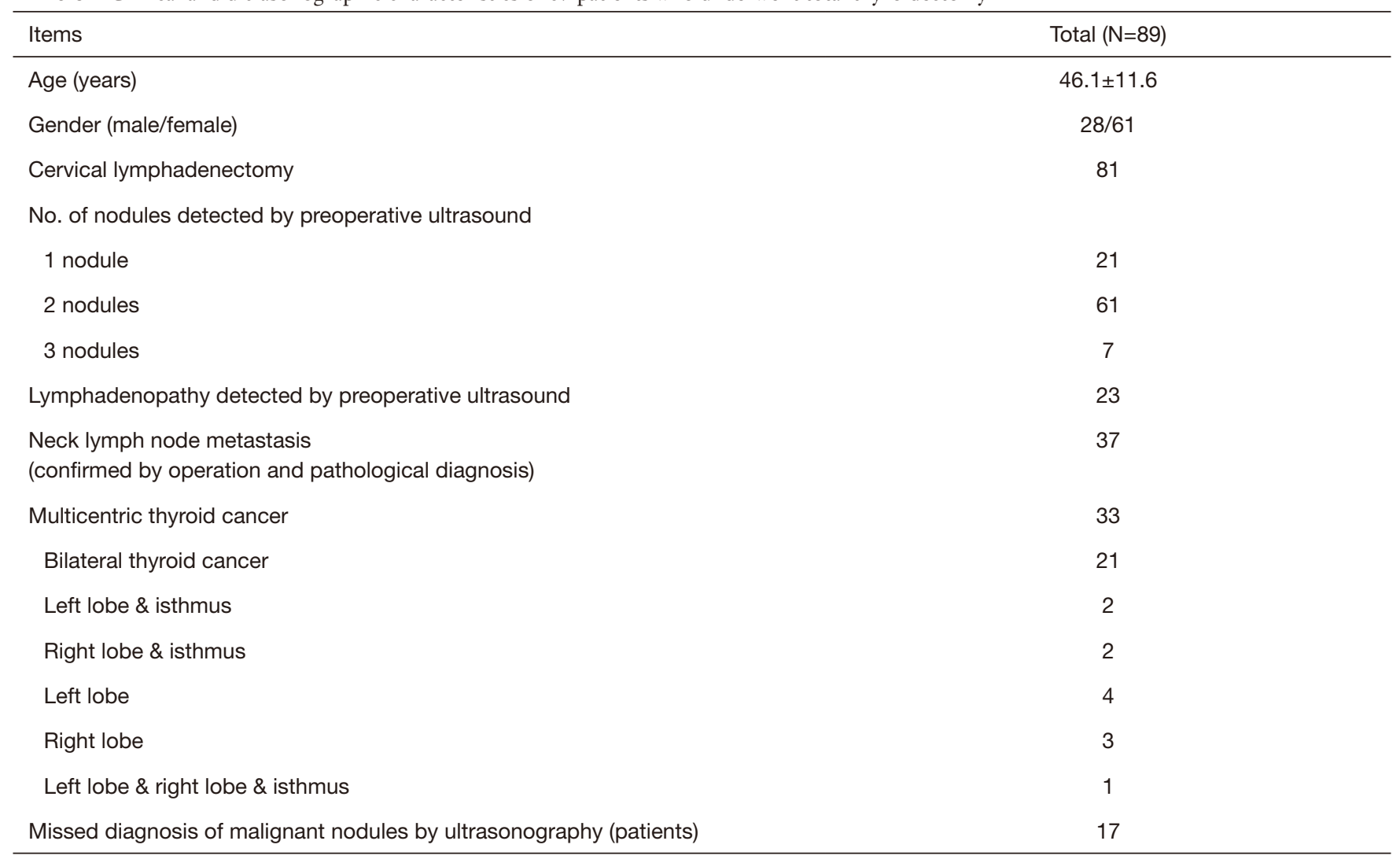

\section{Detection of malignant thyroid nodules by US in patients with multicentric PTC}

Of the 89 patients with PTC, 33 (37.08\%) cases were confirmed as multicentric PTC by operation and pathological examination. Out of the cases of multicentric PTC 22 (66.67\%) were bilateral, 7 were unilateral, and 4 were in the right or left lobe and isthmus. Before surgery, 23 patients were suspected as multicentric PTC by US. Pathological examination revealed that malignant nodules in $17(51.51 \%)$ patients with multicentric PTC had been missed by preoperative US. The malignant nodules that went undetected by US were micronodulars (1-4 $\mathrm{mm})$. In 2 patients, more than 5 micro-nodules $(1-2 \mathrm{~mm})$ were scattered in the thyroid gland. From the 33 patients with multicentric PTC, 17 were confirmed as having lymph node metastasis. The incidence of lymph node metastasis was higher in patients with multicentric PTC than in those with monocentric PTC (17/33 vs. 20/56). However, the difference was not significant. In total, 23 patients were pathologically diagnosed with extracapsular extension, and
9 of these patients were multicentric. Therefore, it can see that there was no association between multicentric PTC and extracapsular extension (9/33 in multicentric PTC vs. 14/56 in monocentric PTC). Characteristics and final diagnoses of 164 thyroid nodules detected by preoperative US were presented in Table 2. Figures 1-5 showed the US imaging of thyroid malignant nodule.

\section{US evaluation of metastatic lymph nodes in the neck and extracapsular extension}

Enlarged neck lymph nodes were detected by preoperative US in 23 patients; however, cervical lymph node metastasis was confirmed by operation and pathological diagnosis in 37 patients. Based on US, 37.84\% of the cases of PTC with metastatic lymph nodes were missed diagnosis. Of the 23 patients with pathologically proven extracapsular extension, US features of extracapsular extension were detected in 19 patients, and 21 patients had cervical lymph node metastasis confirmed by pathology. 
Table 2 Characteristics and final diagnoses of 164 thyroid nodules detected by preoperative US

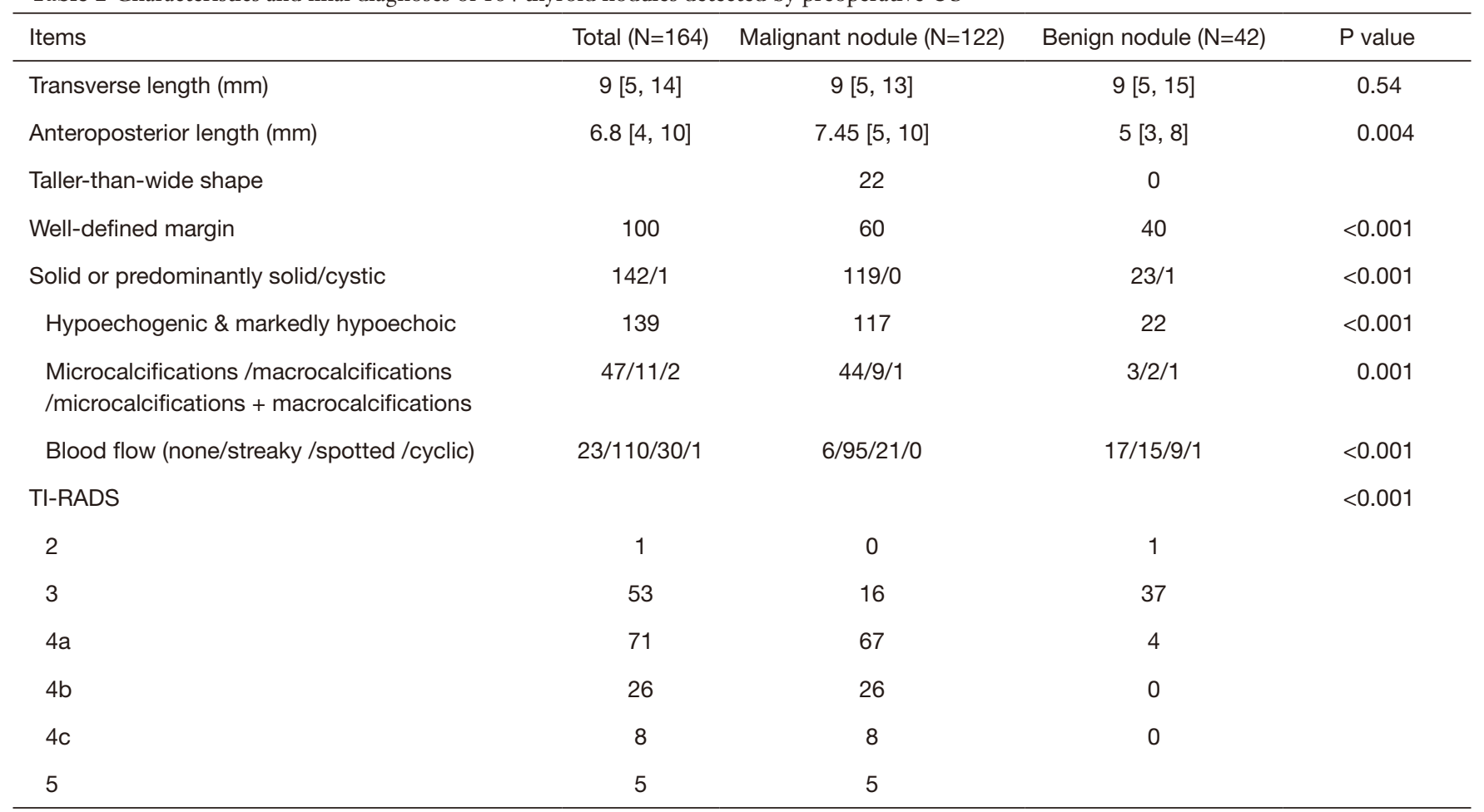

US, ultrasonographic.
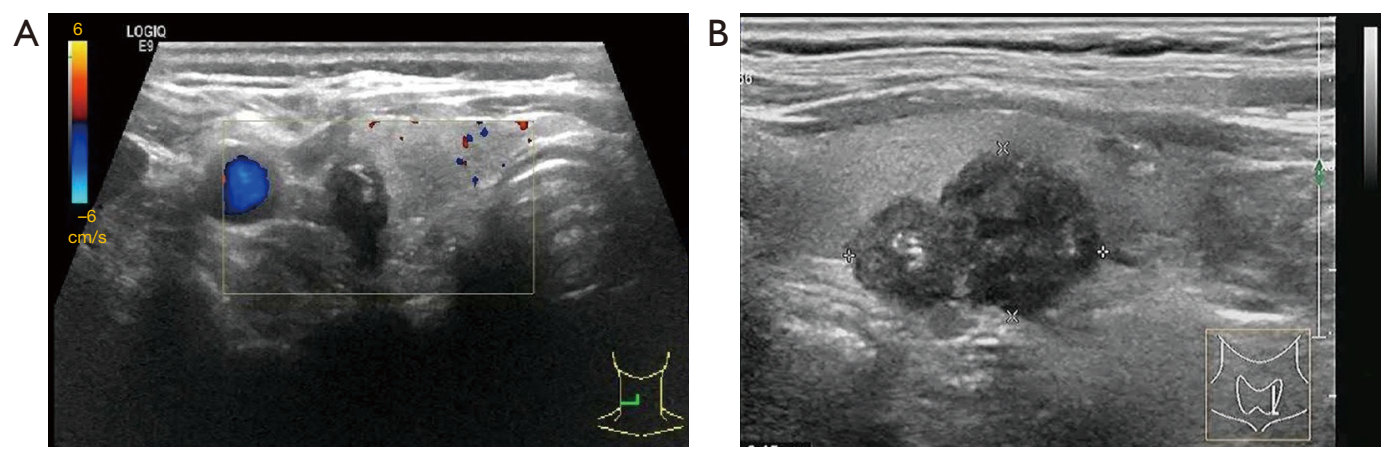

Figure 1 The shape of the malignant nodules. (A) A malignant nodule with taller-than-wide shape; (B) a lobulated malignant nodule. Scattered red spot: blood flow.

\section{Discussion}

In the present study, we retrospectively analyzed data from 89 patients who underwent total thyroidectomy. In total, 164 thyroid nodules were detected by preoperative US. Most patients received FNA before receiving operation. Significantly differences in US pattern were found between benign and malignant nodules. TI-ARDS was used to evaluate the nodules: $87 \%$ of malignant nodules were $4 a-$
5 , while almost $91 \%$ of benign nodules were $2-3$. This indicates that US has good sensitivity and specificity in distinguishing benign and malignant thyroid nodules. In 33 cases with multicentric PTC, only 23 (70\%) patients were suspected as multicentric PTC by preoperative US. The missed malignant nodules were micronodulars (1$4 \mathrm{~mm}$ ). US evaluation is not sensitive enough to detect multicentric thyroid malignant nodules and is also less sensitive in diagnosing neck lymph node metastasis. 

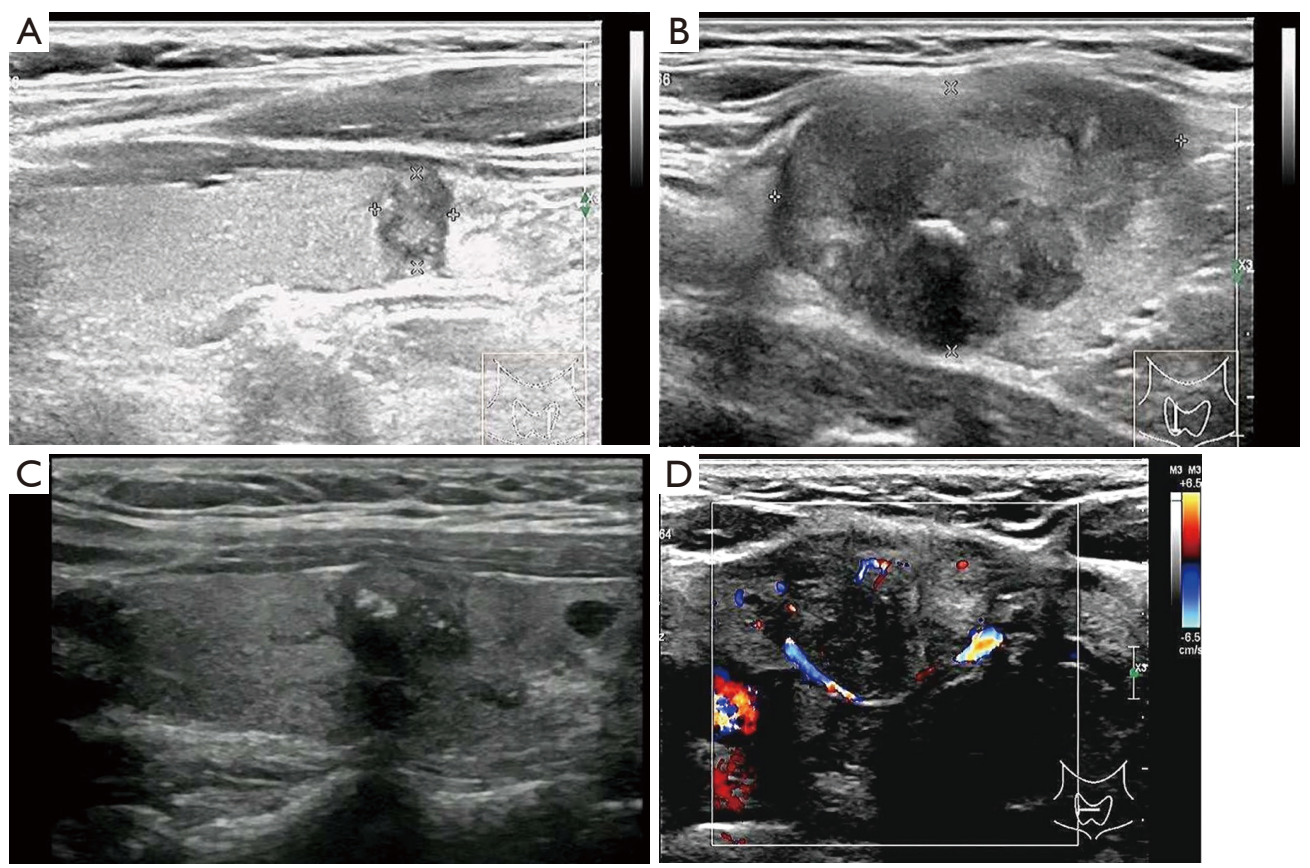

Figure 2 PTC confirmed by pathology and calcifications detected in US. (A) Microcalcification; (B) macrocalcification; (C) microcalcification and macrocalcification; (D) eggshell calcification. PTC, papillary thyroid carcinoma; US, ultrasonographic. Red spot: blood flow.
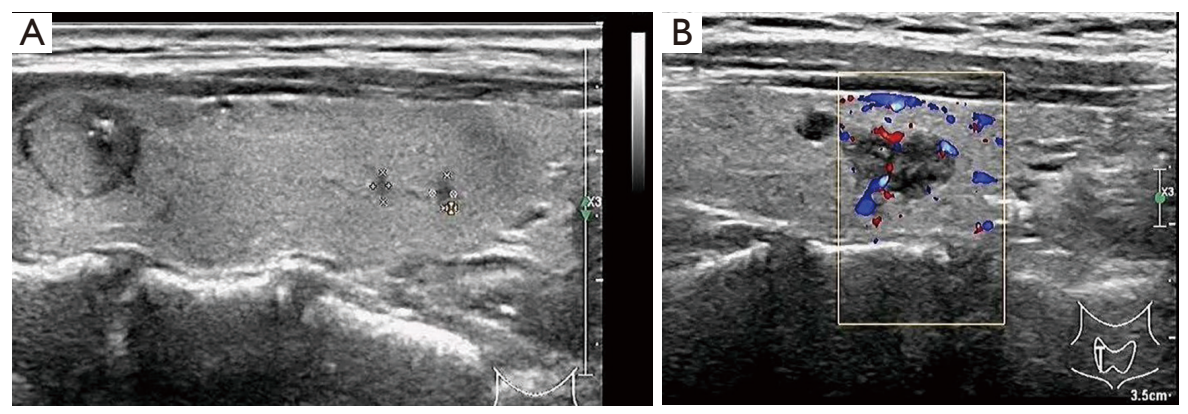

Figure 3 (A) Papillary thyroid microcarcinoma $(1.3 \times 2.3 \mathrm{~mm}$ ) suspected by US and confirmed by pathology; (B) PTC with spotted blood flow (red spot) detected in US. US, ultrasonographic; PTC, papillary thyroid carcinoma.

Thyroid nodules are extremely common. Ultrasonography is the first-line imaging technique for evaluating thyroid nodules (3-5). The role of thyroid sonography is to detect the nodules and determine potential patients who need biopsy $(3-5,13,14)$. Previous studies have proposed that the following multiple sonographic characteristics indicate malignancy: solid or predominantly solid, taller-thanwide shape, hypoechogenicity, poorly defined margins, microcalcifications, increased vascularity, perithyroidal infiltration, and metastatic lymphadenopathy (3-5,19-24).
Purely cystic or spongiform nodules are almost benign (3-5,24). Typical malignant features often appear as a solid hypoechoic nodule with microcalcification, a spiculated/ microlobulated margin, (8). We observed results similar to those of previous studies. Of all 122 malignant nodules, 119 nodules were solid, 117 nodules were hypoechogenic, and vascularity was detected in 116 nodules. However, microcalcification and a spiculated/microlobulated margin were not sensitive for malignant nodules.

Not all US characteristics proposed as indicators of 


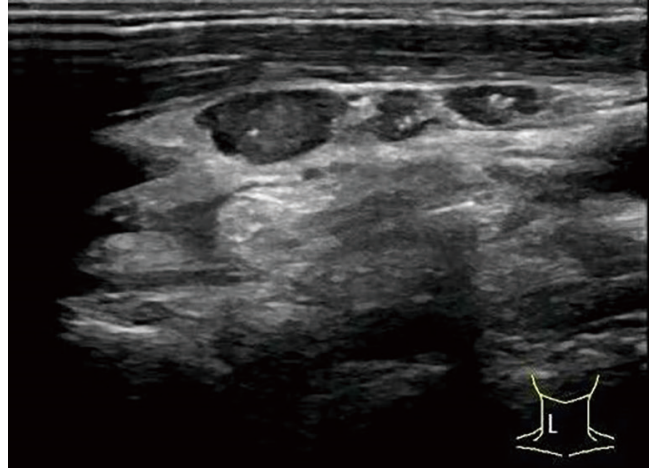

Figure 4 Lymph node metastasis of PTC. PTC, papillary thyroid carcinoma.

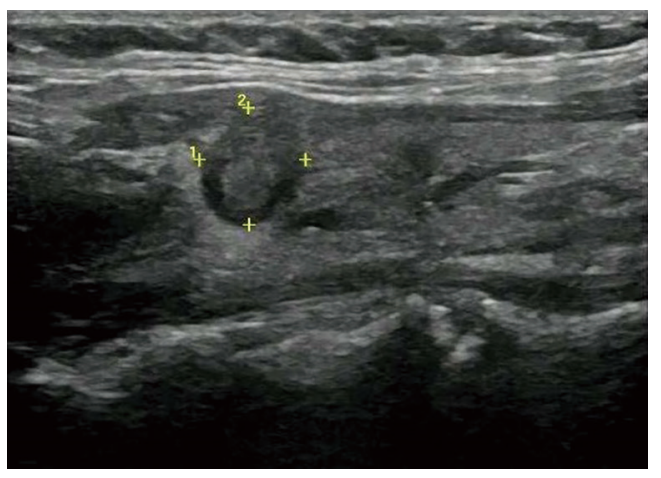

Figure 5 PTC extracapsular extension detected by US. PTC, papillary thyroid carcinoma; US, ultrasonographic.

malignancy have high sensitivity. Cappelli et al. (19) retrospectively analyzed the cytological and histological results of 7,455 nodules. A diagnosis of carcinoma was histologically confirmed in 284 nodules. Microcalcifications, blurred margins, hypoechogenicity, size, and intranodular vascular pattern were associated with malignant nodules (19). However, no single parameter alone is enough to warrant investigation by FNAC (19), they concluded that to achieve balance between missing malignancies and cost benefit, taller-than-wide shape with at least two features out of microcalcification, blurred margins, or hypoechoic pattern is the best compromise (19). Tallerthan-wide shape is a good predictor for malignancy; however, its sensitivity is low. In the present study, only $22(18.03 \%)$ malignant nodules were taller-than-wide shape (when the anteroposterior diameter is longer than the transverse diameter) (19). Microcalcification also offers benefit in predicting malignancy. Several studies have indicated that microcalcifications are more commonly seen in malignant nodules. In Yin et al.'s study (17), the incidence of malignancy was $61.3 \%$ in patients with calcifications, and in Hong et al.'s study (24), microcalcification existed in 59.88\% (194/324) of patients with malignant nodules. Microcalcifications detected by ultrasound may be pathologically related to psammoma bodies in follicular carcinoma (25). Microcalcifications may be a useful prognostic indicator of aggressiveness in PTC (26). In the present study, microcalcifications were detected in 44 (35.77\%) cases with PTC. Therefore, microcalcifications in PTC were less common in the present study than in Yin's study (17). However, in Yin et al.'s study (17), microcalcifications were defined as $<2 \mathrm{~mm}$, which differs from the present study. Other than microcalcifications, macrocalcifications might also serve as an indicator of PCT (16,26-28). In Arpaci et al.'s study, the incidence of malignancy and suspicious for malignancy were higher in nodules with macrocalcification than those without, which suggested that macrocalcifications are not always benign (16). Macrocalcifications, including eggshell macrocalcifications, were detected in 11 patients with PTC in our study, thus supporting the point that macrocalcifications are not always benign.

There is some overlap in the US features of benign and malignant nodules, and not one indicator is perfect. The Thyroid Imaging Reporting and Data System (TIRADS) system integrates these US features (13-15). The ACR TI-RADS allows practitioners to quantify the ultrasound features from a standardized lexicon and give advice for further processing (3-5). According to TI-RADS, TR4 is an indicator of moderately suspicious malignancy, while TR5 implies highly suspicious malignancy (13-15). In the present study, 106 cases with PTC were scored as TR4 or TR5 based on TI-RADS.

In a minority of cases, PTC is highly aggressive, and so identifying high-risk tumors is important in clinical practice. Age, tumor grade, extension, histological grade are all indicators for clinical decision-making. In Džepina et al.'s study (29), the data of 714 patients with PTC were retrospectively analyzed. It was found that 232/669 (34.7\%) of PTCs were multicentric, with foci in the contralateral lobe detected nearly twice as often as in the ipsilateral lobe. In Cheema et al.'s study (30), 23\% of papillary microcarcinomas of the thyroid were multifocal. Some previous studies have supported the inclusion of patients with multicentric tumors in the high-risk group (31). 
Whether multicentric PCT is an indicator of poor prognosis is controversial. Based on data of 1,039 PTC patients, those with multifocal PTC were more prone to have lymph node metastases, more advanced disease, and had higher mortality (32). Multifocality in PTC is a marker of more extensive disease at presentation but is not an independent prognostic factor for long-term outcomes (32). Because multifocality may not be linked to metastatic disease, more extensive surgery may help to improve the outcome for patients with multicentric PTC. According to current clinical guidelines, total or subtotal thyroidectomy is a choice for patients with PTC $<10 \mathrm{~mm}$ based on preoperative comprehensive evaluation (13-15). For multicentric tumors, lobectomy may lead to incomplete surgical treatment, therefore influencing the outcome. Although US is the first-line tool for screening thyroid nodules, data on the use of US for the detection of multicentric PTC is insufficient. In the present study, 33/89 (37.08\%) of PTC cases were multicentric; however, preoperative US missed the diagnosis of malignant nodules in $17(51.51 \%)$ patients with multicentric PTC. US has sufficient sensitivity for detecting thyroid nodules. However, the malignant nodules in these cases were small $(1-3 \mathrm{~mm})$, which may have considerably contributed to the diagnosis being missed in ultrasound evaluation. In two patients, multiple small malignant nodules were scattered in the thyroid tissue. This multiple folic may imply multicentric intrathyroid dissemination (17).

In addition to multicentric tumors, ultrasound is also less sensitive to lymph node metastasis. However, its detection rate for the infiltration of thyroid nodules is excellent. In Kamaya et al.'s study (18), it showed 100\% sensitivity for detecting the presence of capsular abutment and $88 \%$ sensitivity for the detection of extracapsular extension. It had highly specificity but low sensitivity for vascularity beyond the capsule in the diagnosis of extracapsular extension (18). The results of the present study also indicate that US is a useful tool for the detection of extracapsular extension.

The present study has some limitations that need to be addressed. First, it was a single-center, retrospective study with a relatively small sample size compared to those in previous similar studies. In any retrospective study, it is difficult to ensure the consistency of the data, which in this case was US evaluation and measurements by multiple operators. Second, only patients who underwent total thyroidectomy were selected in the analysis of multicentric PCT. We did not include patients with multicentric
PCT who underwent subtotal thyroidectomy. Third, contrast-enhanced sonography, which may be a valuable supplemental method in identifying and distinguishing between benign and malignant thyroid nodules, was not included (33). In the present study, data on contrastenhanced sonography is very limited and only several patients received contrast-enhanced sonography.

Finally, several studies indicated that sonography can predict the BRAF mutational status which is associated with aggressive PCT (9-11). We did not combine the result of BRAF mutation and US to analyze. Combination of contrast-enhanced sonography and BRAF mutation is more sensitive and accurate in detecting malignant nodules and predicting outcome (8-11).

\section{Conclusions}

US evaluation is not sensitive enough to detect multicentric PTC. The minute size of some nodules in multicentric PTC may lead to them being missed by US evaluation. Ultrasonography is an optional tool for the detection of extracapsular extension, but it is less sensitive for diagnosing lymph node metastasis.

\section{Acknowledgments}

Funding: None.

\section{Footnote}

Reporting Checklist: The authors have completed the STROBE reporting checklist. Available at http://dx. doi. org/10. 21037/gs-20-487

Data Sharing Statement: Available at http://dx.doi. org/10.21037/gs-20-487

Conflicts of Interest: Both authors have completed the ICMJE uniform disclosure form (available at http://dx.doi. org/10.21037/gs-20-487). The authors have no conflicts of interest to declare.

Ethical Statement: The authors are accountable for all aspects of the work in ensuring that questions related to the accuracy or integrity of any part of the work are appropriately investigated and resolved. This study has been approved by the hospital's ethics committee (No. 2018032). Informed consent was taken from all the patients. The study 
was conducted in strict accordance with the Declaration of Helsinki of the World Medical Association.

Open Access Statement: This is an Open Access article distributed in accordance with the Creative Commons Attribution-NonCommercial-NoDerivs 4.0 International License (CC BY-NC-ND 4.0), which permits the noncommercial replication and distribution of the article with the strict proviso that no changes or edits are made and the original work is properly cited (including links to both the formal publication through the relevant DOI and the license). See: https://creativecommons.org/licenses/by-nc-nd/4.0/.

\section{References}

1. Leboulleux S, Tuttle RM, Pacini F, et al. Papillary thyroid microcarcinoma: time to shift from surgery to active surveillance? Lancet Diabetes Endocrinol 2016;4:933-42.

2. Ullmann TM, Gray KD, Moore MD, et al. Current controversies and future directions in the diagnosis and management of differentiated thyroid cancers. Gland Surg 2018;7:473-86.

3. Andrioli M, Valcavi R. Sonography of Normal and Abnormal Thyroid and Parathyroid Glands. Front Horm Res 2016;45:1-15.

4. Li Q, Lin X, Shao Y, et al. Imaging and screening of thyroid cancer. Radiol Clin N Am 2017;55:1261-71.

5. Wong R, Farrell SG, Grossmann M. Thyroid nodules: diagnosis and management. Med J Aust 2018;209:92-8.

6. Amin MB, Edge SB, Greene F, et al, editors. AJCC Cancer Staging Manual, 8th ed. New York, NY: Springer International Publishing, 2017.

7. Hundahl SA, Fleming ID, Fremgen AM, et al. A National Cancer Data Base report on 53,856 cases of thyroid carcinoma treated in the U.S., 1985-1995 [see commetns]. Cancer 1998;83:2638-48.

8. Shin JH. Ultrasonographic imaging of papillary thyroid carcinoma variants. Ultrasonography 2017,36:103-10.

9. Lee JH, Lee ES, Kim YS. Clinicopathologic significance of BRAF V600E mutation in papillary carcinomas of the thyroid: a meta-analysis. Cancer 2007;110:38-46.

10. Chen L, Chen L, Liu J, et al. The Association Among Quantitative Contrast-Enhanced Ultrasonography Features, Thyroid Imaging Reporting and Data System and BRAF V600E Mutation Status in Patients With Papillary Thyroid Microcarcinoma. Ultrasound Q 2019;35:228-32.

11. Lin ZM, Yan CX, Song Y, et al. The features of contrast enhanced ultrasound and BRAF V600E in papillary thyroid carcinoma. J Thorac Dis 2019;11:5071-8.

12. Lin JD, Chao TC, Hsueh C, et al. High recurrent rate of multicentric papillary thyroid carcinoma. Ann Surg Oncol 2009;16:2609-16.

13. Haugen BR, Alexander EK, Bible KC, et al. 2015 American Thyroid Association Management Guidelines for Adult Patients with Thyroid Nodules and Differentiated Thyroid Cancer: The American Thyroid Association Guidelines Task Force on Thyroid Nodules and Differentiated Thyroid Cancer. Thyroid 2016;26:1-133.

14. Horvath E, Silva CF, Majlis S, et al. Prospective validation of the ultrasound based TIRADS (Thyroid Imaging Reporting And Data System) classification: results in surgically resected thyroid nodules. Eur Radiol 2017;27:2619-28.

15. Kim EK, Park CS, Chung WY, et al. New sonographic criteria for recommending fine-needle aspiration biopsy of nonpalpable solid nodules of the thyroid. AJR Am J Roentgenol 2002;178:687-91.

16. Arpaci D, Ozdemir D, Cuhaci N, et al. Evaluation of cytopathological findings in thyroid nodules with macrocalcification: macrocalcification is not innocent as it seems. Arq Bras Endocrinol Metabol 2014;58:939-45.

17. Yin L, Zhang W, Bai W, et al. Relationship Between Morphologic Characteristics of Ultrasonic Calcification in Thyroid Nodules and Thyroid Carcinoma. Ultrasound Med Biol 2020;46:20-5.

18. Kamaya A, Tahvildari AM, Patel BN, et al. Sonographic Detection of Extracapsular Extension in Papillary Thyroid Cancer. J Ultrasound Med 2015;34:2225-30.

19. Cappelli C, Castellano M, Pirola I, et al.Thyroid nodule shape suggests malignancy. Eur J Endocrinol 2006;155:27-31.

20. Hoang JK, Lee WK, Lee M, et al. US Features of thyroid malignancy: pearls and pitfalls. Radiographics 2007;27:847-60.

21. Cappelli C, Castellano M, Pirola I, et al. The predictive value of ultrasound findings in the management of thyroid nodules. QJM 2007;100:29-35.

22. Remonti LR, Kramer CK, Leitão CB, et al. Thyroid ultrasound features and risk of carcinoma: a systematic review and meta-analysis of observational studies. Thyroid 2015;25:538-50.

23. Tessler FN, Middleton WD, Grant EG, et al. ACR Thyroid Imaging, Reporting and Data System (TI-RADS): White Paper of the ACR TI-RADS committee. J Am Coll 
Radiol 2017;14:587-95.

24. Hong HS, Lee JY. Diagnostic Performance of Ultrasound Patterns by K-TIRADS and 2015 ATA Guidelines in Risk Stratification of Thyroid Nodules and Follicular Lesions of Undetermined Significance. AJR Am J Roentgenol 2019;213:444-50.

25. Kim BK, Lee EM, Kim JH, et al. Relationship between ultrasonographic and pathologic calcification patterns in papillary thyroid cancer. Medicine 2018;97:e12675.

26. Yoon DY, Lee JW, Chang SK, et al. Peripheral calcification in thyroid nodules: ultrasonographic features and prediction of malignancy. J Ultrasound Med 2007;26:1349-55.

27. Kim BM, Kim MJ, Kim EK, et al. Sonographic differentiation of thyroid nodules with eggshell calcifications. J Ultrasound Med 2008;27:1425-30.

28. Kim BK, Choi YS, Kwon HJ, et al. Relationship between patterns of calcification in thyroid nodules and histopathologic findings. Endocr J 2013;60:155-60.

29. Džepina D, Zurak K, Petric V, et al. Pathological

Cite this article as: Lu C, Wang Y, Yu M. Is ultrasonographic evaluation sensitive enough to detect multicentric papillary thyroid carcinoma? Gland Surg 2020;9(3):737-746. doi: 10.21037/gs-20-487 characteristics and clinical perspectives of papillary thyroid cancer: study of 714 patients. Eur Arch Otorhinolaryngol 2014;271:141-8.

30. Cheema Y, Olson S, Elson D, et al. What is the biology and optimal treatment for papillary microcarcinoma of the thyroid? J Surg Res 2006;134:160-2.

31. Leboulleux S, Rubino C, Baudin E, et al. Prognostic factors for persistent or recurrent disease of papillary thyroid carcinoma with neck lymph node metastases and/ or tumor extension beyond the thyroid capsule at initial diagnosis. J Clin Endocrinol Metab 2005;90:5723-9.

32. Geron Y, Benbassat C, Shteinshneider M, et al. Multifocality Is not an Independent Prognostic Factor in Papillary Thyroid Cancer: A Propensity Score-Matching Analysis. Thyroid 2019;29:513-22.

33. Ma X, Zhang B, Ling W. Contrast-Enhanced Sonography for the Identification of Benign and Malignant Thyroid Nodules: Systematic Review and Meta-Analysis. J Clin Ultrasound 2016;44:199-209. 
Supplementary

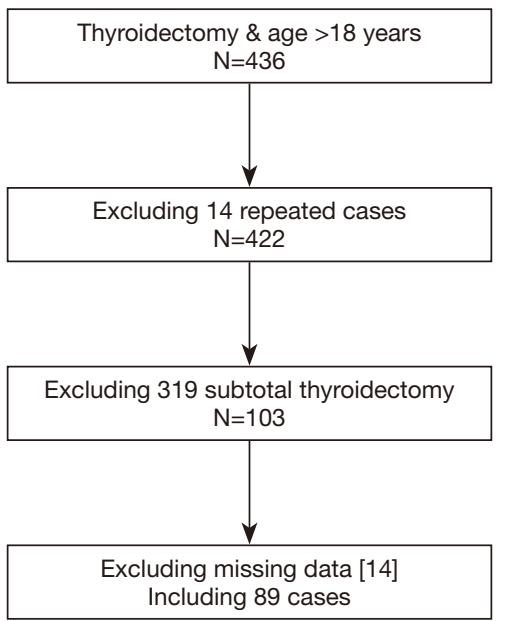

Figure S1 Flow chart of this study. 